\title{
Final Technical Report, DOE/ER/64323
}

June 5, 2013

ID:ER64323-1029401-0012964

Principal Investigator: Albert J. Valocchi 217-333-3176

Co-PIs:

Institution: University of Illinois Board of Trustees

Title: Modeling Multiscale-Multiphase-Multicomponent Subsurface Reactive Flows Using Advanced Computing

SC Division:SC-23.4

Program Manager: Robert T. Anderson 301-903-5549

\section{INTRODUCTION}

Professor Albert J. Valocchi, at the University of Illinois, was part of a multiinstitution SciDAC-funded team that developed PFLOTRAN, the next-generation ("petascale') massively parallel, multiphase, multicomponent reactive flow and transport code. These codes are required to improve understanding and risk management of subsurface radionuclide migration and geological sequestration of carbon dioxide. The overall project was led by Los Alamos National Laboratory and included Argonne, Oak Ridge and Pacific Northwest National Laboratories, in addition to the University of Illinois.

The official project performance period was October 2006 through September 2011. Due to administrative delays, The University of Illinois did not officially receive funding until February 2007. A post-doctoral research associate worked on the project from June 12007 to Dec 31, 2008, when he left to become an assistant professor at Texas A\&M University. A new doctoral student started on the project August 15, 2008. A second Masters student started working on the project in January 2012. Both students will complete their degrees in August 2013. Due to the delay in starting the project, the early departure of the post-doc for a faculty position, and partial fellowship support for the graduate students, the project was granted a no-cost extension until May 14, 2013.

The overall broad research tasks assigned to the University of Illinois are upscaling sub-grid-scale variability and implementation of full tensor permeability and dispersion. Most conventional numerical methods that include full tensor permeability/dispersion do not guarantee positive solution for arbitrary unstructured grids. Due to the nonlinearity of the multiphase flow and transport equations, negative values for fluid pressure and solute concentration are not tolerable and result in code malfunction.

An additional research thrust that we have pursued is the use of complex multiphase flow and transport models for uncertainty and risk analysis related to $\mathrm{CO}_{2}$ sequestration. We have worked on methods for global sensitivity analysis, which aims to identify which input parameters have the most important impact on uncertain response of 
the model. This allows development of simp0lified 'meta-models' that can be used in lieu of the computationally burdensome PFLOTRAN model for risk assessment.

\section{RESULTS - FULL TENSOR DISPERSION}

Permeability is one of the most important parameters that affect multi-phase fluid flow in the subsurface. Permeability is a tensor having principal directions determined by small-scale geological structure. When we simulate large-scale complex subsurface formations, it is not possible to always align the numerical grid with the principal directions of anisotropy, and hence it is necessary to consider full-tensor permeability in our numerical implementation.

Hydrodynamic dispersion is one of the most important parameters that affect the transport of constituents in the subsurface. Dispersion governs the rate of spreading and mixing caused by small-scale heterogeneities, and will play a critical role in controlling the dissipation of the rising buoyant $\mathrm{CO} 2$ plume and the mixing of species for geochemical reactions. Hydrodynamic dispersion is a tensor with principal directions controlled by the direction of flow. Therefore, it is impossible to align the numerical grid with the principal directions since they will change with space and time in response to hydrologic boundary conditions and inputs (e.g., pumping) even for a uniform porous medium.

When standard discretization methods are used for full tensors, it is well-known that non-physical oscillations can result. In fact, this problem can even occur for scalar diffusion if the numerical mesh does not satisfy certain geometrical properties. A numerical method that suffers from this problem is referred to as giving "non-monotonic" solutions or as violating the "discrete maximum principle" or DMP. For reactive transport simulations this problem can be potentially disastrous, since negative values of solute concentration will result and the code can crash when these concentrations are used in highly nonlinear reaction equations. This is particularly relevant for PFLOTRAN, which uses a tightly coupled global implicit formulation. Therefore, many numerical models of reactive transport (e.g., TOUGHREACT, STOMP) simply ignore off-diagonal terms in the dispersion tensor or assume scalar dispersion.

There has been much research over the years in developing new methods and adapting existing methods to guarantee monotonic solutions under the widest possible set of conditions. Some recent work for finite element methods is reported by (Kuzmin and Shashkov, 2009) and (Nakshatrala and Valocchi, 2009). The most well-known methods to address monotonicity for finite volume methods are the multi-point flux approximations (Aavatsmark, 2002; Nordbotten and Aavatsmark, 2007); although these methods work well under many conditions, they are not proven to always guarantee a positive solution. A recent finite volume formulation that is proven to be monotone is by Lipnikov and co-workers at Los Alamos (Lipnikov et al., 2009). 
The existing structure of PFLOTRAN is based upon standard finite volume discretizations, and hence it would require some re-programming to implement the monotone methods by Lipnikov and co-workers. There is another strategy based upon the classical flux corrected transport (FCT) ideas of (Boris and Book 1973) and (Zalesak 1979). A unified algebraic-based framework has been developed by Kuzmin and coworkers (Kuzmin and Turek 2002), and we have implemented and tested this for a simplied model problem. The appeal of this method is that it is algebraic, and is thus relatively straightforward to introduce into an existing code. The reference by Kuzmin can be consulted for details about the algorithm and the theory. The general idea is that the overall transport matrix is separated into a low-order part that is monotone and a remaining term that represents the additional high-order fluxes. At each time step, a limiting strategy is employed at each grid block to only add in enough of the additional high-order flux to avoid introducing oscillations. It can be demonstrated that the limiting strategy leads to a final result (referred to as the "limited solution") that preserves mass balance.

To demonstrate the performance of the FCT approach, we present the results of a reactive transport test problem reported in Garcia-Cabrejo and Valocchi (2012a). A square pulse of chemical A is initially placed in the center of a square domain containing contaminant B. There is a spatially varying flow field that results from a heterogeneous hydraulic conductivity field, and hence there is a spatially varying dispersion tensor.

Dispersion will cause the contaminants $\mathrm{A}$ and $\mathrm{B}$ to mix and react according to the simple bi-molecular reaction: $\mathrm{A}+\mathrm{B} \rightarrow \mathrm{C}$. A standard second-order finite volume discretization is used. The results shown below in Figure 1 demonstrate that the FCT approach can be effective in eliminating the negative concentrations of reactant $\mathrm{A}$ and the product $\mathrm{C}$ (indicated in the white regions of the left figure). Here the negative concentrations result in non-physical results; however, we emphasize that in more complex non-linear reactive transport problems, the negative concentrations may cause the code to crash.
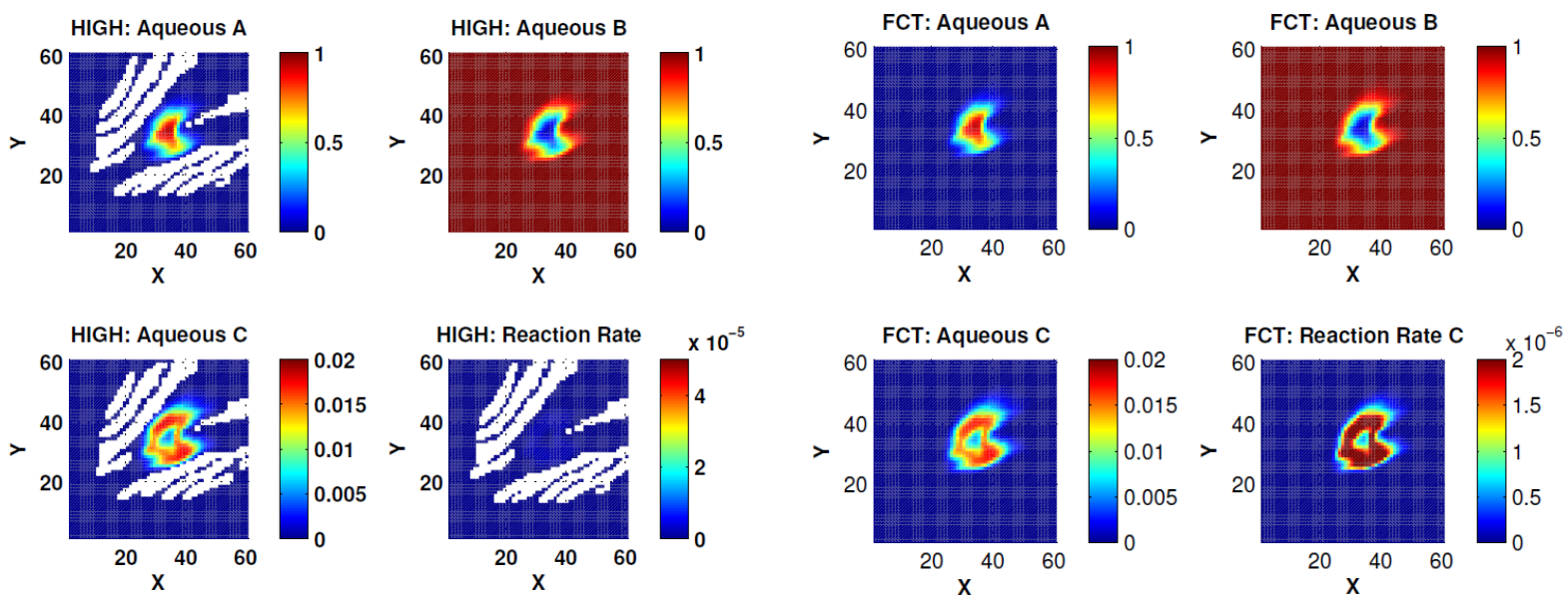

Figure 1. Use of FCT approach with conventional finite volume discretization of fulltensor dispersion in reactive transport: $\mathrm{A}+\mathrm{B} \rightarrow \mathrm{C}$ 
Although the FCT approach is appealing due to its algebraic nature and flexibility to be used with a wide variety of discretization methods, it has some disadvantages. The process for calculating the flux limiter leads to a non-linear method; the correction factors should be computed at every iteration of the solution of the reactive transport problem and this will increase computation time. The method also leads to a lower-order truncation error. To address these shortcomings, we have also worked on a more rigorous approach for achieving non-negative solutions when finite element discretization is used. This work is reported by Nakshatrala and Valocchi (2009) and Nakshatrala et al. (2013). The basic idea behind the method is to take advantage of the variational theory of the finite element method, namely that although discrete solution (for the simple case of a linear non-reactive solute) requires solution of a system of linear algebraic equations, the solution can also be posed as an optimization problem. Therefore, it is conceptually straightforward to add constraints to guarantee certain physical properties of the solution (i.e., non-negative solution). To date we have only addressed diffusion problems, since in this case the resulting optimization problem is simple to solve since it falls into the class of convex quadratic programming. We are working on extending to include advection.

To illustrate the method, we show a result reported in Nakshatrala et al. (2013). The problem is similar to that shown above in Figure 1. An initial pulse of reactant $A$ is in the center of a domain containing reactant $\mathrm{B}$. There is anisotropic dispersion oriented at a 45 degree angle to the horizontal. As the two reactant mix, product $\mathrm{C}$ is formed by the same reaction as in Figure 1. The standard Galerkin method clearly gives negative and non-physical values for the product $\mathrm{C}$, while our optimization-base method works.
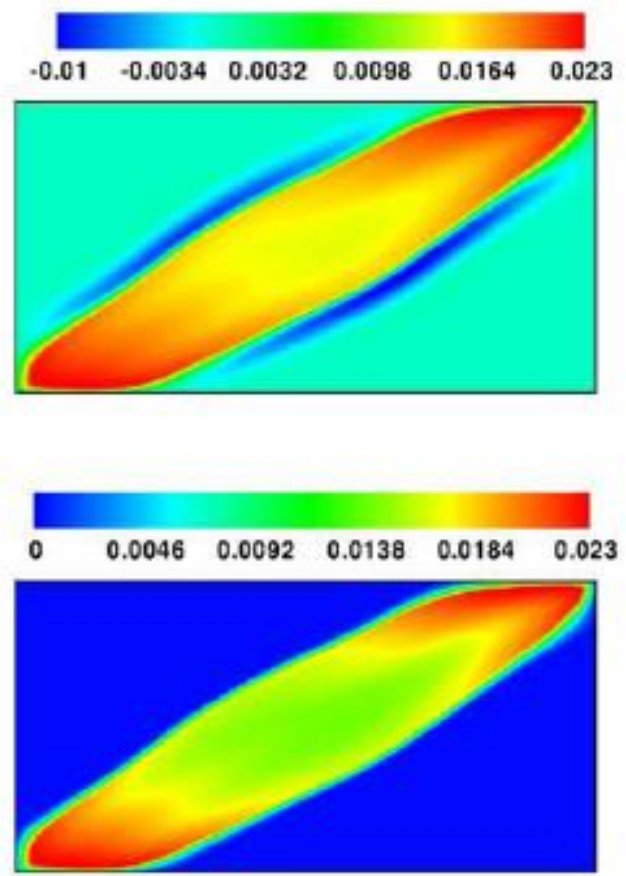

Figure 2. Diffusion and reaction of an initial slug: This figure shows the contours of the concentration of the product $\mathrm{C}$ obtained using the Galerkin formulation (top figure), and the proposed computational framework (bottom figure) at $\mathrm{t}=$ $0.05 \mathrm{~s}$. As one can see from the figure, the Galerkin formulation violated the non-negative constraint. On the other hand, the proposed computational framework satisfies the non-negative constraint for the concentration of the product $\mathrm{C}$. 


\section{RESULTS - UNCERTAINTY QUANTIFICATION FOR CO2 SEQUESTRATION}

The transport and fate of $\mathrm{CO} 2$ depends upon many interacting nonlinear processes; these physics are built into numerical models like PFLOTRAN. There are many hydrogeological and physical parameters that need to be specified prior to running a model like PFLOTRAN, and all of these parameters are subject to great uncertainty. We have been working in the area of Global Sensitivity Analysis (GSA) using the approaches pioneered by Saltelli and co-workers (Saltelli and Ratto, 2008); these methods attribute the total uncertainty in the model output to various input parameters and can be used to rank the importance of input parameters. Using the results of GSA, we can develop reduced-order "meta-models" that give a computationally efficient representation of the numerical model; we use the so-called Polynomial Chaos Expansion (Ghanem and Spanos, 1991). Our results will be fully reported in the doctoral thesis of O. GarciaCabrejo, to be completed in August 2013. Preliminary results are in Garcia-Cabrejo and Valocchi (2012b), and Garcia-Cabrejo and Valocchi (2013a,b,c)

\section{References:}

Aavatsmark, I. (2002). An introduction to multipoint flux approximations for quadrilateral grids, Computational Geosciences, 6, 405-432.

Boris, J. and Book, D. (1973). Flux-corrected transport. I. SHASTA, a fluid transport algorithm that works. Journal of Computational Physics, 11(1), 38-69.

Garcia-Cabrejo, O, and Albert Valocchi, Application of flux corrected transport for multicomponent reactive transport modeling, CMWR2012: XIX International Conference on Computational Methods in Water Resources, University of Illinois, June 17-21, 2012a. http://cmwr2012.cee.illinois.edu

Garcia-Cabrejo, O, and Albert Valocchi, Sensitivity analysis in numerical simulation of multiphase flow for $\mathrm{CO} 2$ storage in saline aquifers using the probabilistic collocation approach, CMWR2012: XIX International Conference on Computational Methods in Water Resources, University of Illinois, June 17-21, 2012b. http://cmwr2012.cee.illinois.edu

Garcia-Cabrejo, O, and Albert Valocchi, Global Sensitivity Analysis for Multivariate Output using Polynomial Chaos Expansion, Reliability Engineering \& System Safety, submitted, 2013a.

Garcia-Cabrejo, O, and Albert Valocchi, Influence of the parameters of the input PDF in the probability of leakage of $\mathrm{CO} 2$ through abandoned wells, International Journal Of Greenhouse Gas Control., in preparation, 2013b.

Garcia-Cabrejo, O, and Albert Valocchi, Global sensitivity analysis of storage mechanisms of $\mathrm{CO} 2$ in saline aquifers: effect of the correlation of input and output, Advances in Water Resources, in preparation, 2013c. 
Ghanem, R. and Spanos, P. (1991). Stochastic Finite Elements: A Spectral Approach. Dover Publication. ISBN 978-0486428185.

Kuzmin, D. and S. Turek (2002). "Flux Correction Tools for Finite Elements." Journal of Computational Physics 175(2): 525-558.

Kuzmin, D. and Shashkov, M. (2009). A constrained finite element method satisfying the discrete maximum principle for anisotropic diffusion problems. Journal of Computational Physics

Lipnikov, K., D. Svyatskiy, and Y. Vassilevski. Interpolation-free monotone finite volume method for diffusion equations on polygonal meshes. Journal of Computational Physics, 228:703-716, 2009.

Nakshatrala, K. and A.J. Valocchi, Non-negative mixed finite element formulations for a tensorial diffusion equation, J. Comput. Physics, doi: DOI: 10.1016/j.jcp.2009.05.039, 228, 6726-6752, 2009.

Nakshatrala, K., M.K. Mudunuru, and A.J. Valocchi, A numerical framework for diffusion-controlled bimolecular-reactive systems to enforce maximum principles and the non-negative constraint, Journ Comput Physics, revised manuscript under review, 2013.

Nordbotten, J. and Aavatsmark, I. (2007). Monotonicity of control volume methods. Numerische Mathematik, 106, 255-288.

Saltelli, A. and Ratto, M. (2008). Global sensitivity analysis: the primer. WileyInterscience.

Zalesak, S. (1979). Fully multidimensional flux-corrected transport algorithms for fluids. Journal of Computational Physics, 31(3), 335-362.

\section{PRODUCTS DELIVERED}

Five manuscripts published:

(1) Nakshatrala, K. and A.J. Valocchi, Non-negative mixed finite element formulations for a tensorial diffusion equation, J. Comput. Physics, doi: DOI: 10.1016/j.jcp.2009.05.039, 228, 6726-6752, 2009.

(2) Herrera, P., A.J. Valocchi, and R.D. Beckie, A multidimensional streamline-based method to simulate reactive solute transport in heterogeneous porous media, $A d v$. Water Resour., 33(7), 711-727, 2010 
(3) Nakshatrala, K. and A.J. Valocchi, Variational structure of the optimal artificial diffusion method for the advection-diffusion equation, International Journal of Computational Mechanics, 7, 559-572, 2010.

(4) Turner, D. Z., Nakshatrala, K. B. and Hjelmstad, K. D. (2011), A stabilized formulation for the advection-diffusion equation using the Generalized Finite Element Method. International Journal for Numerical Methods in Fluids, 66: 64-81. doi: 10.1002/fld.2248

(5) Valocchi, A.J., Hydrogeochemical Models, Chapter 4 in Delivery and Mixing in the Subsurface: Processes and Design Principles for In Situ Remediation, P.K. Kitanidis and P.L. McCarty, eds., DOD SERDP/ESTCP Monograph Series, Springer, 2012.

Two manuscripts submitted:

(1) Nakshatrala, K., M.K. Mudunuru, and A.J. Valocchi, A numerical framework for diffusion-controlled bimolecular-reactive systems to enforce maximum principles and the non-negative constraint, Journal of Computational Physics, revision under review, 2013.

(2) Garcia-Cabrejo, O, and Albert Valocchi, Global Sensitivity Analysis for Multivariate Output using Polynomial Chaos Expansion, Reliability Engineering \& System Safety, submitted, 2013.

Eight conference presentations/proceedings:

(1) "Investigation of Numerical Upscaling Techniques for Mixing-Controlled Reactions in Heterogeneous Media,” K. Nakshatrala, A.J. Valocchi, AGU Fall Meeting, San Francisco, Dec. 2007.

(2) "A survey on non-negative numerical formulations for tensorial diffusion equations," K. Nakshatrala, A.J. Valocchi, Computational Methods in Water Resources, XVII International Conference,San Francisco, July 6-11, 2008.

(3) "A multiscale finite element framework for simulating reactive contaminant transport in heterogeneous porous media," K. Nakshatrala, A.J. Valocchi, Computational Methods in Water Resources, XVII International Conference,San Francisco, July 6-11, 2008.

(4) K. Nakshatrala and A.J. Valocchi, A Multiscale Finite Element Framework For Reactive Contaminant Transport In Heterogeneous Porous Media, IMECE200867395, Proceedings of ASME IMECE 2008 ASME Mechanical Engineering Congress and Exposition October 31 - November 6, 2008, Boston, USA.

(5) "Reactions along Transverse Mixing Zones in Heterogeneous Aquifers: Using Effective Dispersion Coefficients and Multi-Scale Finite Element Methods," A.J. Valocchi, K.B. Nakshatrala, AGU Fall Meeting, San Francisco, Dec. 2009. 
(6) Garcia-Cabrejo, O. and A.J. Valocchi “Application of flux-corrected transport for multicomponent reactive transport," SIAM Conference on Computational and Mathematical Issues in the Geosciences, Long Beach, March 21-24, 2011.

(7) Garcia-Cabrejo, O, and Albert Valocchi, Application of flux corrected transport for multicomponent reactive transport modeling, CMWR2012: XIX International Conference on Computational Methods in Water Resources, University of Illinois, June 17-21, 2012a. http://cmwr2012.cee.illinois.edu

(8) Garcia-Cabrejo, O, and Albert Valocchi, Sensitivity analysis in numerical simulation of multiphase flow for $\mathrm{CO} 2$ storage in saline aquifers using the probabilistic collocation approach, CMWR2012: XIX International Conference on Computational Methods in Water Resources, University of Illinois, June 17-21, 2012b. http://cmwr2012.cee.illinois.edu 